\title{
A multi-phase model for simulations of galaxy formation
}

\author{
Volker Springel \\ Max-Planck-Institute for Astrophysics, Karl-Schwarzschild-Str. 1, 85740 \\ Garching, Germany
}

\section{Lars Hernquist}

Harvard-Smithsonian Center for Astrophysics, 60 Garden Street, Cambridge, MA 01238, USA

\begin{abstract}
We discuss SPH simulations of galaxy formation which use a hybrid method to describe a two-phase structure of the star forming ISM on unresolved scales. Our modeling includes radiative cooling, heating due to a UV background, growth of cold clouds embedded in an ambient hot gas, star formation out of cloud material, feedback due to supernovae in the form of thermal heating and cloud evaporation, starbursts that can lead to galactic outflows, and metal enrichment. Our particular model for the treatment of the two-phase structure is based on a modified and extended version of the grid-based approach of Yepes et al. (1997). We discuss the properties of the feedback model and show how it stabilizes star forming disk galaxies and reduces the cosmic star formation rate to a level consistent with current observational constraints.
\end{abstract}

\section{Introduction}

Cosmological simulations of galaxy formation that include radiative cooling of gas and simple phenomenological models for star formation typically exhibit an overproduction of stars compared to observational constraints. Part of this 'overcooling' problem may be caused by low-resolution effects of the commonly employed SPH technique, but even when corrected, cooling appears to be too efficient, particularly in small halos at high redshift. It is thus commonly believed that feedback processes associated with supernovae explosions, stellar winds, or UV radiation, play an important role in regulating star formation, and need to be properly taken into account for a successful model of galaxy formation.

However, attempts to include feedback into cosmological simulations have proven to be difficult and only shown limited success (among others: Katz, Weinberg \& Hernquist, 1996; Thacker \& Couchman, 2000; Kay et al., 2001). Pure thermal feedback has been demonstrated to be ineffective, and direct attempts to include kinetic feedback are not easy to justify physically. In general, the shape of the luminosity function of galaxies has not been fit successfully, especially at the faint end, and the feedback schemes have not been able to account for outflows, which are presumably occurring in starbursting galaxies at high redshift. 
It appears increasingly unlikely that these difficulties can be overcome by simple parameter changes or slight alterations of the current generation of modeling techniques. Instead, true progress may require that the physics of star formation and feedback is modeled more faithfully within cosmological simulations. In particular, the observed multi-phase structure of the interstellar medium (ISM) may represent a key phenomenon which should not be neglected in numerical studies of galaxy formation.

However, it is clear that brute-force attempts to resolve the true spatial structure of the ISM within cosmological volumes will remain computationally prohibitive in the near future. It may however be possible to include some of the relevant physics as a sub-grid model for individual fluid elements. In this contribution, we extend a multi-phase approach for the ISM that was suggested in a similar form by Yepes et al. (1997) for a Eulerian grid code (see also Hultman $\&$ Pharasyn, 1999). Its properties make it an interesting model that may cure many of the problems encountered by current single-phase simulations. We will first define the model in detail, and then highlight some of its properties using star forming spiral galaxies as examples. We will also briefly discuss some of its implications for cosmological simulations of galaxy formation.

\section{The model}

We call our model for star formation and feedback a 'hybrid' technique because it does not explicitly resolve the spatial multi-phase structure of the ISM on small scales, but rather makes the assumption that essential aspects of the global dynamical behavior of the ISM can be described in terms of an effective sub-grid model that only uses spatially averaged properties to describe the medium.

In this hybrid approach, each SPH particle is taken to represent a patch of the ISM, which we here picture as a co-spatial fluid of condensed cold clouds embedded at pressure equilibrium in an ambient hot gas. The hybrid particles may also have a component of stars, forming out of the clouds. For these hybridparticles, the equations of hydrodynamics are only followed for the ambient medium. The cold clouds and the stars are subject to gravity, add inertia, and participate in mass and energy exchange processes with the ambient gas phase. These processes are computed on a particle by particle basis in terms of simple differential equations for a highly simplified model for the physics of the ISM. The specific model described here tries to incorporate some of the key aspects of the theoretical picture of the ISM outlined by McKee \& Ostriker (1977).

In the following, $\rho_{h}$ represents the local density of the ambient gas, $\rho_{c}$ is the density of cold clouds, $\rho_{\star}$ the density of stars, and $\rho=\rho_{h}+\rho_{c}$ denotes the total gas density. Because individual molecular clouds and stars cannot be resolved, $\rho_{c}$ and $\rho_{\star}$ are taken to be local averages over a small region of the ISM, which is represented by one SPH particle.

We model three basic processes that lead to mass exchange between the phases. These are star formation, cloud evaporation due to supernovae, and cloud growth due to cooling. We discuss these processes in turn, focusing first on self-regulated, 'quiescent' star formation. Later, we will extend the model to include an additional run-away (i.e. 'starburst') mode of star formation. For simplicity, we will omit adiabatic terms in the following definition of the model. 
We assume that star formation converts cold clouds into stars on a timescale $t_{\star}$, and for each portion of stars formed, a fraction $\beta$ is taken to be short-lived and to explode as supernovae instantly. This can be described by:

$$
\frac{\mathrm{d} \rho_{\star}}{\mathrm{d} t}=\frac{\rho_{c}}{t_{\star}}-\beta \frac{\rho_{c}}{t_{\star}}=(1-\beta) \frac{\rho_{c}}{t_{\star}} .
$$

Star formation therefore leads to a depletion of the cold reservoir at the rate $\rho_{c} / t_{\star}$, and to an increase of the mass in the ambient phase as $\beta \rho_{c} / t_{\star}$, where we assume that ejecta from supernovae are returned to the smooth gaseous phase. The parameter $\beta$ is the mass fraction of massive stars $\left(Z 8 M_{\odot}\right)$ formed for each initial population of stars and hence depends on the adopted initial mass function (IMF).

Apart from returning gas (enriched with metals) to the ambient phase of the ISM, the supernovae also release energy. The exact value of the available energy depends on the IMF. For the canonical value of $10^{51}$ ergs per supernova and the IMF adopted here, we expect an average return of $\epsilon_{\mathrm{SN}}=4 \times 10^{48} \mathrm{ergs} \mathrm{M}_{\odot}^{-1}$ for each solar mass in stars formed. The heating rate due to supernovae is hence

$$
\left.\frac{\mathrm{d}}{\mathrm{d} t}(\rho u)\right|_{\mathrm{SN}}=\epsilon_{\mathrm{SN}} \frac{\mathrm{d} \rho_{\star}}{\mathrm{d} t}=\beta u_{\mathrm{SN}} \frac{\rho_{c}}{t_{\star}},
$$

where $u_{\mathrm{SN}} \equiv(1-\beta) \beta^{-1} \epsilon_{\mathrm{SN}}$ may be expressed in terms of an equivalent 'supernova temperature' $T_{\mathrm{SN}}=2 \mu u_{\mathrm{SN}} /(3 k) \simeq 10^{8} \mathrm{~K}$.

We assume that the supernova feedback energy is directly heating the ambient hot phase. In addition, we assume that cold clouds are evaporated inside the hot bubbles of exploding supernovae, essentially by thermal conduction, thereby returning material from condensed clouds to the ambient phase. We take the total mass of clouds that are evaporated to be proportional to the mass in supernovae themselves, viz.

$$
\left.\frac{\mathrm{d} \rho_{c}}{\mathrm{~d} t}\right|_{\mathrm{EV}}=A \beta \frac{\rho_{c}}{t_{\star}} .
$$

The efficiency $A$ of the evaporation process is expected to be a function of the local environment. For simplicity, we will only take the expected theoretical dependence on density, $A \propto \rho^{-4 / 5}$, into account (McKee \& Ostriker, 1977). We will not try to estimate the evaporation efficiency from first principles, but rather treat its normalization as a parameter. As we will discuss below, the range of permissible values for $A$ is limited once we require a plausible temperature structure for the ISM model.

Finally, we invoke a process that lets cold clouds grow and come into existence in the first place. We here assume that a thermal instability is operating in the region of coexistence between the cold and hot phases, leading to mass exchange between ambient gas and cold clouds. In particular, we assume that the energy radiated away cools material from the temperature of the hot phase to that of the cold phase, leading to a growth of the mass contained in clouds. This mass flux is thus:

$$
\left.\frac{\mathrm{d} \rho}{\mathrm{d} t}\right|_{\mathrm{TI}}=\frac{1}{u_{h}-u_{c}} \Lambda_{\mathrm{net}}\left(\rho_{h}, u_{h}\right)
$$


Here $u_{h}$ and $u_{c}$ are the internal energies per unit mass for hot and cold phases, respectively. We will typically assume a temperature of $T_{c} \simeq 8000 \mathrm{~K}$ for the cold clouds. Of course, real clouds will form cores that are much colder, for example by molecular cooling processes, but this is not important for the model presented here, where we only rely on $u_{h} \gg u_{c}$. Adopting smaller values for $u_{c}$ would not influence our results. In the following, we will use a fudge factor

$$
f= \begin{cases}1 & \text { normal cooling } \\ 0 & \text { thermal instability active }\end{cases}
$$

to differentiate between situations where the thermal instability is assumed to be operating, and regions where it is ineffective. In general, the gas can be expected to exhibit thermally unstable behavior if the cooling rate is a falling function of temperature. For a primordial mixture of gas this is the case roughly in the range $10^{5}-10^{6} \mathrm{~K}$, while for metal-enriched gas the minimum of the cooling curve shifts to higher temperatures, up to a a few times $10^{7} \mathrm{~K}$. We thus require that the ISM temperature lies within this range. For the onset of the thermal instability itself, we chose a simple density threshold criterion, with $f=0$ for $\rho>\rho_{\text {th }}$, and $f=1$ otherwise. This is motivated by the observed threshold behavior of star formation (Kennicutt, 1983, 1989).

Summarizing the above, the mass transfer rates for hot and cold phases can be written as:

$$
\begin{aligned}
& \frac{\mathrm{d} \rho_{c}}{\mathrm{~d} t}=-\frac{\rho_{c}}{t_{\star}}-A \beta \frac{\rho_{c}}{t_{\star}}+\frac{1-f}{u_{h}-u_{c}} \Lambda_{\text {net }}\left(\rho_{h}, u_{h}\right) \\
& \frac{\mathrm{d} \rho_{h}}{\mathrm{~d} t}=\beta \frac{\rho_{c}}{t_{\star}}+A \beta \frac{\rho_{c}}{t_{\star}}-\frac{1-f}{u_{h}-u_{c}} \Lambda_{\text {net }}\left(\rho_{h}, u_{h}\right)
\end{aligned}
$$

In both of these equations, the first terms on the right hand side describe star formation and feedback, the second cloud evaporation, and the third the growth of clouds due to radiative cooling of gas.

We now discuss the energy budget of the gas. Let $\epsilon=\rho_{h} u_{h}+\rho_{c} u_{c}$ denote the total energy per unit volume of the gas, where $u_{h}$ and $u_{c}$ are the energy per unit mass for hot and cold components. We can write the energy budget as:

$$
\frac{\mathrm{d}}{\mathrm{d} t}\left(\rho_{h} u_{h}+\rho_{c} u_{c}\right)=-\Lambda_{\mathrm{net}}\left(\rho_{h}, u_{h}\right)+\beta \frac{\rho_{c}}{t_{\star}} u_{\mathrm{SN}}-(1-\beta) \frac{\rho_{c}}{t_{\star}} u_{c}
$$

where $\Lambda_{\text {net }}$ is the radiative cooling function of the ambient hot medium. Only the ambient gas is assumed to undergo radiative processes. The second term describes the non-gravitational energy injection by exploding supernovae. Finally, the third term gives the loss of energy in the gaseous phase due to material that is locked up in collisionless stars. For this loss term we assume that the material that is converted into stars is at the temperature of the cold clouds.

Using our model assumptions, we can split the energy equation into two separate equations for the thermal budget of hot and cold components:

$$
\begin{gathered}
\frac{\mathrm{d}}{\mathrm{d} t}\left(\rho_{c} u_{c}\right)=-\frac{\rho_{c}}{t_{\star}} u_{c}-A \beta \frac{\rho_{c}}{t_{\star}} u_{c}+\frac{(1-f) u_{c}}{u_{h}-u_{c}} \Lambda_{\text {net }} \\
\frac{\mathrm{d}}{\mathrm{d} t}\left(\rho_{h} u_{h}\right)=\beta \frac{\rho_{c}}{t_{\star}}\left(u_{\mathrm{SN}}+u_{c}\right)+A \beta \frac{\rho_{c}}{t_{\star}} u_{c}-\frac{u_{h}-f u_{c}}{u_{h}-u_{c}} \Lambda_{\text {net }}
\end{gathered}
$$



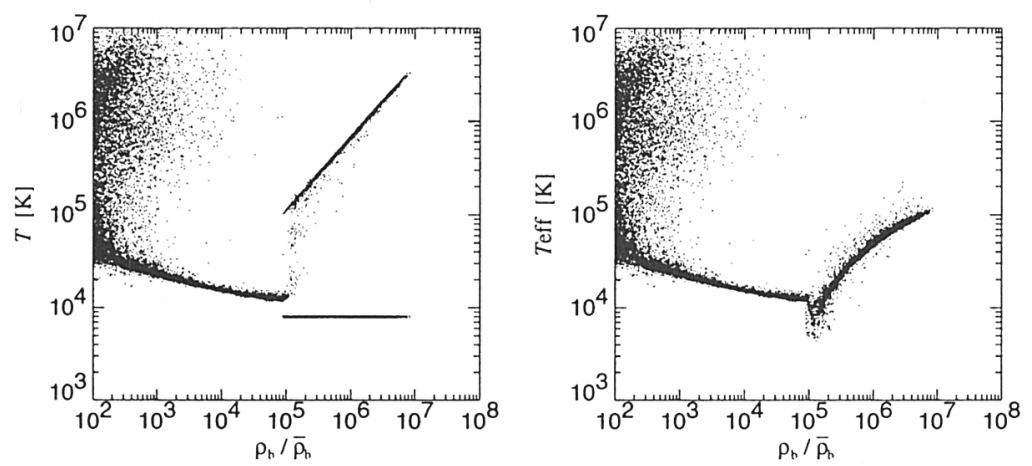

Figure 1. Temperature structure (left) and effective pressure (right) of the two-phase medium as a function of density in a cosmological simulation. Above overdensities of $\simeq 10^{5}$, the ISM contains clouds at $8000 \mathrm{~K}$ embedded in a ambient hot phase with a temperature that rises with density. The plume of gas on the left of the diagrams is shock heated gas in halos. On the right, the pressure is shown in terms of an effective temperature $T_{\mathrm{eff}}=\mu P_{\mathrm{eff}} /(k \rho)$.

Note that we will assume that the cold clouds are at a fixed temperature, so $u_{c}$ will be treated as a constant. As a consequence, the above equations give the evolution of the temperature of the hot phase as

$$
\rho_{h} \frac{\mathrm{d} u_{h}}{\mathrm{~d} t}=\beta \frac{\rho_{c}}{t_{\star}}\left(u_{\mathrm{SN}}+u_{c}-u_{h}\right)-A \beta \frac{\rho_{c}}{t_{\star}}\left(u_{h}-u_{c}\right)-f \Lambda_{\text {net }} .
$$

This is one of the fundamental equations that is integrated in the simulation code, augmented of course with a term coming from the external work by pressure forces. Actually, for purely numerical reasons (Springel \& Hernquist, 2001) we prefer to use an equivalent formulation of the above equation in terms of specific entropy.

A closer inspection of equation (11) reveals some interesting properties. Suppose the thermal instability is operative $(f=0)$, then the temperature of the hot phase changes only due to star formation and feedback processes. In fact, as long as there is star formation, the temperature will evolve towards

$$
u_{h}=\frac{u_{\mathrm{SN}}}{A+1}+u_{c}
$$

This means that provided star formation is rapid enough compared to adiabatic changes of temperature due to the motion of the gas, the temperature of the hot phase will be maintained at the value set by equation (12), which is independent of the star formation timescale $t_{\star}$. Since usually $A \gg 1$ and $u_{\mathrm{SN}} / A \gg u_{c}$ this temperature is in practice just given by $u_{h} \simeq u_{\mathrm{SN}} / A$. For a supernova temperature of $10^{8} \mathrm{~K}$ and, say, $A=100$, the self-regulated temperature of the hot diffuse phase of the ISM will thus be maintained at $\simeq 10^{6} \mathrm{~K}$.

A further interesting consequence of the above feedback model is that it leads to self-regulation of star formation. Due to the evaporation process, star 
formation acts to reduce the density in cold clouds, thus lowering the star formation rate. On the other hand, a higher density of hot gas leads to an increase of the cooling rate, and hence to faster replenishing of clouds, thereby acting to increase the star formation rate. In this way, a self-regulation cycle of star formation is established where in dynamical equilibrium the growth of clouds is balanced by their evaporation due to supernova feedback.

This condition can be seen in more detail by considering equation (8). In the self-regulated regime of star formation, we expect the effective pressure

$$
P_{\text {eff }}=(\gamma-1)\left(\rho_{h} u_{h}+\rho_{c} u_{c}\right)
$$

of the medium to be constant in time. This condition implies

$$
\frac{\rho_{c}}{t_{\star}}=\frac{\Lambda_{\text {net }}\left(\rho_{h}, u_{h}\right)}{\beta u_{\mathrm{SN}}-(1-\beta) u_{c}} .
$$

Using $\Lambda_{\text {net }}\left(\rho_{h}, u_{h}\right)=\Lambda\left(u_{h}\right) \rho_{h}^{2}$ and $\beta u_{\text {SN }} \gg u_{c}$, we thus obtain an expression for the expected ratio of mass in the cold and hot phases, viz. $\rho_{c} / \rho_{h}=$ $t_{\star} \Lambda\left(u_{h}\right) \rho_{h} /\left(\beta u_{\mathrm{SN}}\right)$. Once the thermal instability sets in, we expect the cold clouds to be the dominant component in mass, i.e. $\rho_{h} \ll \rho_{c}$ and $\rho \simeq \rho_{c}$. We can then estimate the mass fraction of the hot component as

$$
\left(\frac{\rho_{h}}{\rho_{c}}\right)^{2}=\frac{\beta u_{\mathrm{SN}}}{t_{\star} \Lambda\left(u_{h}\right) \rho_{c}} \simeq \frac{\beta u_{\mathrm{SN}}}{t_{\star} \Lambda\left(u_{\mathrm{SN}} / A\right) \rho} .
$$

The effective pressure of the gas will therefore take on the value

$$
P_{\mathrm{eff}}=(\gamma-1) \rho\left\{\frac{u_{\mathrm{SN}}}{A}\left[\frac{\beta u_{\mathrm{SN}}}{t_{\star} \rho \Lambda\left(u_{\mathrm{SN}} / A\right)}\right]^{1 / 2}+u_{c}\right\} .
$$

If the first term in the curly brackets becomes larger than $u_{c}$, the medium will thus be pressurized by star formation. The dependence of $P_{\text {eff }}$ on density is particularly important for the dynamical stability of star forming regions and can provide enough vertical thickening to a gaseous disk to prevent rapid break-up into clumps due to dynamical instabilities. In Figure 1, we show the temperature structure that results for this model in star forming regions, and how it leads to pressurization of the medium.

\section{Parameter choices}

We parameterize the density dependence of the supernova evaporation parameter as

$$
A(\rho)=A_{0}\left(\frac{\rho}{\rho_{\mathrm{th}}}\right)^{-4 / 5},
$$

where $\rho_{\mathrm{th}}$ and $A_{0}$ are parameters of the model. We also need to specify the star formation timescale $t_{\star}$. We here follow a common assumption often adopted in cosmological SPH simulations by making the star formation timescale proportional to the local dynamical time of the gas. We thus set

$$
t_{\star}(\rho)=t_{0}^{\star}\left(\frac{\rho}{\rho_{\mathrm{th}}}\right)^{-1 / 2}
$$




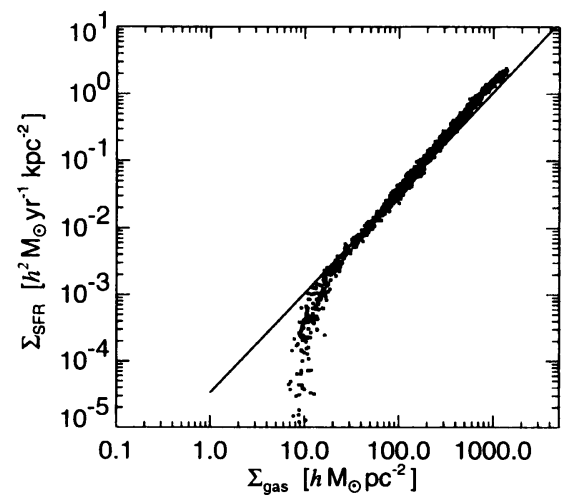

Figure 2. Star formation rate per unit area versus gas surface density in a quiescently star forming disk galaxy. The symbols show simulation measurements at various outputs times for a run with $t_{0}^{\star}=6 \mathrm{Gyr}$. The solid line indicates the 'Kennicutt'-law.

where $t_{0}^{\star}$ is a parameter of the model. For the purposes of this work, we neglect uncertainties in the IMF and treat $\beta$ and $u_{\mathrm{SN}}$ as known constants. However, the three parameters $\rho_{\mathrm{th}}, A_{0}$, and $t_{0}^{\star}$ are of crucial importance for the behavior of the model and should ideally be either eliminated, or tightly constrained by observations.

To obtain a plausible model, we first require that at the onset of the thermal instability, the equilibrium temperature of the hot medium is such that the thermal instability in fact becomes operative. This means that the cooling function should start to fall at this temperature, which happens at $\simeq 10^{5} \mathrm{~K}$. We thus require that $T_{\mathrm{SN}} / A_{0}=10^{5} \mathrm{~K}$, which fixes $A_{0}$ to a value of $\simeq 1000$. Next, we argue that self-regulation of star formation can only work if the pressure generated by it is dynamically relevant at all. In the context of the present model, this may be expressed by requiring that the first term in the curly brackets of equation (16) reaches at least the size of $u_{c}$. Assuming that this condition just holds at the onset of star formation, we have

$$
\rho_{\mathrm{th}}=\left(\frac{u_{\mathrm{SN}}}{A_{0} u_{c}}\right)^{2} \frac{\beta u_{\mathrm{SN}}}{t_{0}^{\star} \Lambda\left(u_{\mathrm{SN}} / A_{0}\right)} .
$$

Given a value for $t_{0}^{\star}$, the density threshold $\rho_{\text {th }}$ is then determined. The model therefore depends on one real free parameter, the star formation timescale $t_{0}^{\star}$. Note that this parameter sensitively determines the star formation rates obtained in simulations that use this model. We can hence use the observed star formation rates of local disk galaxies (Kennicutt, 1983, 1989) to determine $t_{0}^{\star}$. The observed 'Kennicutt'-law consists of a power-law correlation

$$
\Sigma_{\mathrm{SFR}}=(2.5 \pm 0.7) \times 10^{-4}\left(\frac{\Sigma_{\text {gas }}}{\mathrm{M}_{\odot} \mathrm{pc}^{-2}}\right)^{1.4 \pm 0.15} \frac{\mathrm{M}_{\odot}}{\mathrm{yrkpc}^{2}}
$$

between the average star formation rate per unit area and the surface gas density, and a cut-off for star formation activity below a density of approximately $\Sigma_{\text {gas }} \simeq$ 
$10 \mathrm{M}_{\odot} \mathrm{pc}^{-2}$. In Figure 2, we show simulation results for the star formation densities obtained for isolated disk galaxies, where a value of $t_{0}^{\star}=6 \mathrm{Gyr}$ was used in order to match the observational amplitude of star formation. Note that matching the slope is non-trivial, because it requires that the vertical structure that develops under the action of $P_{\text {eff }}$ for a self-gravitating disk of gas leads to star formation rates per unit area which are compatible with the observed Kennicutt-law. Also note that the cut-off obtained in the simulation is at the right place. In the present model, the cut-off for the star formation rate is induced by the value chosen for $\rho_{\text {th }}$, which however depends on $t_{0}^{\star}$. It is thus a nice consistency check for the model that the implied cut-off matches the observed one once $t_{0}^{\star}$ is set to reproduce the amplitude of the Kennicutt-law.

\section{Starbursts}

As described above, the multi-phase model is effective in establishing a numerically well controlled regulation cycle for the star formation of gas that has cooled and collapsed to very high baryonic overdensities. Gas contained in dark matter halos can thus cool and settle into a rotationally supported disk where the baryons are gradually turned into stars, with a rate that is in accordance with observations of disk galaxies. However, the model will not be able to account in this form for the rich phenomenology associated with starbursts and galactic outflows. These energetic events are likely to be of crucial importance to understand the enrichment of the intergalactic medium (IGM) with metals, and the apparent 'preheating' of the gas observed in the intracluster medium. Also, a process that can reheat and transport collapsed material from the center of a galaxy into or out of its extended dark matter halo seems to be required to reproduce both the shape and amplitude of the observed cosmic star formation history. This is also suggested by semi-analytic models of galaxy formation which need to invoke such feedback processes to arrive at a successful galaxy formation scenario.

To account for starbursts, we assume that the self-regulation of star formation breaks down at sufficiently high gas densities, being replaced by an 'explosive' (i.e. very rapid) mode of star formation. In the self-regulated regime, cold clouds are imagined to be constantly formed and evaporated. In fact, if clouds are not replenished by cooling, they would be consumed on a timescale

$$
t_{c}=\frac{t_{\star}}{\beta A}=\frac{t_{0}^{\star}}{\beta A_{0}}\left(\frac{\rho}{\rho_{\mathrm{th}}}\right)^{3 / 10} .
$$

This timescale $t_{c}$ describes the speed on which clouds are reprocessed. At the onset of star formation, it is about a factor $\sim 100$ shorter than the star formation timescale itself, on the order a few times $10^{7}$ years. However, for higher densities, clouds are reprocessed more slowly, and eventually $t_{c}$ will become larger than the maximum lifetime of individual clouds. At this point it is becoming unlikely that tight self-regulation can be maintained. Instead we will assume that all of the clouds begin to collapse and to form stars, i.e. the timescale of star formation will suddenly become very short.

Since it is rather unclear how this transition to accelerated star formation proceeds in detail, we model it in a simple way. We assume that self-regulation 


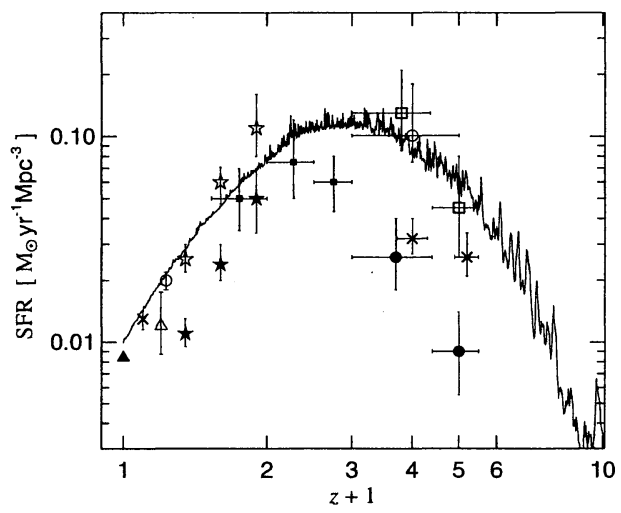

Figure 3. Star formation rate per unit volume in a cosmological simulation using the multi-phase model including starbursts. Symbols show various observational constraints. In this simulation, only about $10 \%$ of all the baryons are locked up in stars at $z=0$.

breaks down once the evaporation parameter falls below a prescribed value $A_{\mathrm{sb}}$, at which point we will assume rapid star formation with $t_{\star} \rightarrow 0$. The existence of this threshold imposes an upper bound on the cloud reprocessing timescale, and also a maximum temperature for the self-regulated hot phase of the ISM.

If the starburst threshold $A_{\mathrm{sb}}$ is reached for a particle with mass $M_{c}$ in cold clouds, this mass will then be turned into stars and hot supernova remnants with masses $M_{\star}=M_{c}(1-\beta) /\left(1+\beta A^{\prime}\right)$ and $M_{h}=\beta M_{c}\left(1+A^{\prime}\right) /\left(1+\beta A^{\prime}\right)$, and the temperature of the remnant gas will be set to $u_{r}=u_{\mathrm{SN}} /\left(1+A^{\prime}\right)+u_{c}$. This assumes that even in this 'burst' mode of star formation, cloud evaporation takes place with efficiency $A^{\prime}$. It is plausible that $A^{\prime}$ is substantially smaller than $A_{\mathrm{sb}}$, because many clouds will already have turned into stars once the supernovae begin exploding. On the other hand, it is unlikely that evaporation would be completely suppressed. Lacking better estimates, we here assume $A^{\prime}=10$ for this process, which will roughly split the cold cloud mass equally in stars and hot remnant gas, with the latter ending up at a temperature of about $10^{7} \mathrm{~K}$. For $A_{\mathrm{sb}}$ we select a value of 25 for now, but we note that we in principle allow this value to be adjusted in order to improve fits of cosmological simulations to observations. In particular, we expect that $A_{\mathrm{sb}}$ will have a strong impact on the cosmic star formation history, the metal enrichment of the IGM, and the faint end of the luminosity function, among others. Any of these observations may thus be used to constrain $A_{\text {sb }}$.

Once a starburst event in the above sense has taken place, we create a new star particle of mass $M_{\star}$, and the hybrid particle that is left behind is reduced in mass to $M_{h}$ (plus any hot gas it had before the event), all of it being in the ambient phase, i.e. the particle becomes a 'normal' single-phase gas particle at this point. After a time-delay of $10^{7}$ years, corresponding to the lifetime of massive stars, all of the supernova energy produced in the starburst is injected into the gas particle that is left behind. This triggers a small explosion, and in general accelerates the particle due to the created pressure forces towards the negative density gradient. In a star forming disk, the collective effect of many 
of such star-bursting particles in the central region can then produce a bipolar galactic wind. For small enough galaxies, the gas can actually leave the dark halo entirely and become gravitationally unbound from it.

Using this starburst model, we obtain a very good match between observational constraints on the star formation history and simulation results. In Figure 3, we show a result for a small cosmological test simulation with $2 \times 100^{3}$ particles. Starbursts are very frequent at high redshift, and become increasingly less important at low redshift. Outflowing material actually enriches and heats the IGM. We will give a more detailed analysis of these results in future work.

\section{Conclusions}

While the modeling of star formation and feedback discussed here is still phenomenological to a large degree, we think it is arguably better motivated by the underlying physics than feedback schemes previously employed in numerical studies of galaxy formation on cosmological scales. The ability of the model to simultaneously match the star formation law of isolated disk galaxies and the cosmic star formation history make it a highly promising candidate for further simulation work on galaxy formation. In particular, the approach accounts for galactic outflows and will thus allow us to address a large number of highly interesting questions related to the enrichment and heating of the IGM, and its relation to star forming high- $z$ galaxies. An SPH approach like the one presented here seems almost ideally suited to study these problems. Alternatively, adaptive mesh refinement codes may also be fruitfully combined with a sub-grid technique like the one discussed here.

We have already applied an earlier version of the current model in a large simulation of the 'concordance' cosmology, using $2 \times 300^{3}$ particles in a box $33.5 h^{-1} \mathrm{Mpc}$ on a side. First results for this particular run, which did not yet account for starbursts and metal enrichment, have been discussed by White, Hernquist \& Springel (2001a,b). In future work, we plan to investigate the consequences of starbursts for galaxy formation and the IGM in detail, using simulations of comparable or even larger size.

\section{References}

Hultman, J., \& Pharasyn, A. 1999, A\&A, 347, 769

Katz, N., Weinberg, D. H., \& Hernquist, L. 1996, ApJS, 105, 19

Kay, S. T., Pearce, F. R., \& Frenk, C. S., Jenkins, A. 2001, astro-ph/0106462

Kennicutt, R. C. 1983, ApJ, 272, 54

Kennicutt, R. C. 1989, ApJ, 344, 685

McKee, C. F., \& Ostriker, J. P. 1977, ApJ, 218, 148

Springel, V., \& Hernquist, L. 2001, astro-ph/0111016

Thacker, R. J., \& Couchman, H. M. P. 2000, ApJ, 545, 728

White, M., Hernquist, L., \& Springel, V. 2001a, ApJ, 550, L129

White, M., Hernquist, L., \& Springel, V. 2001b, astro-ph/0107023

Yepes, G., Kates, R., Khokhlov, A., \& Klypin, A. 1997, MNRAS, 284, 235 